\title{
Review Article \\ Stereotypes of Aging: Their Effects on the Health of Older Adults
}

\author{
Rylee A. Dionigi \\ School of Human Movement Studies, Charles Sturt University, Bathurst, NSW 2795, Australia \\ Correspondence should be addressed to Rylee A. Dionigi; rdionigi@csu.edu.au \\ Received 5 August 2015; Revised 13 October 2015; Accepted 25 October 2015 \\ Academic Editor: Tiffany F. Hughes
}

Copyright (C) 2015 Rylee A. Dionigi. This is an open access article distributed under the Creative Commons Attribution License, which permits unrestricted use, distribution, and reproduction in any medium, provided the original work is properly cited.

\begin{abstract}
The purpose of this review is to present findings on the effects of stereotypes of aging on health outcomes related to older adults, such as physical and mental functioning (specifically) and overall well-being and perceived quality of life (more broadly). This review shows that both positive and negative stereotypes of aging can have enabling and constraining effects on the actions, performance, decisions, attitudes, and, consequently, holistic health of an older adult. This review further highlights a variety of limitations in stereotype research in aging contexts, including a lack of qualitative studies focusing on older adult perspectives and the fluctuating definition of what constitutes "good health" during older age.
\end{abstract}

\section{Introduction}

Aging is a highly individualized and complex process; yet it continues to be stereotyped, especially in Western cultures. Stereotypes about a particular group play a powerful role in shaping how we think about and interact with individuals, as well as how individuals within the stereotyped group see themselves [1].

Stereotypes are unchallenged myths or overstated beliefs associated with a category which are widespread and entrenched in verbal, written, and visual contexts within society [2]. Stereotypes of aging include assumptions and generalizations about how people at or over a certain age should behave, and what they are likely to experience, without regard for individual differences or unique circumstances [3].

Stereotypes of aging in contemporary culture, particularly North America, are primarily negative, depicting later life as a time of ill health, loneliness, dependency, and poor physical and mental functioning $[1,3]$. However, stereotypes of ageing can also be positive (e.g., healthy, wealthy, and wise) or neutral and they are continually changing over time and across contexts [3-5]. Views of old age, and the perceptions older adults hold of themselves, are complex, multidimensional, and dynamic [5]. In other words, stereotypes of aging are social constructs that are culturally and historically situated, as well as individually interpreted.
Notably, any stereotype of aging (including those that equate aging with frailty and decline, or later life with health and affluence) has the potential to reinforce ageism (i.e., social oppression based on age $[6,7])$ because they position ill health in old age as undesirable $[8,9]$ and they do not acknowledge the vast diversity among older adults [3]. The terms "older adults," "older people," "older individuals," "old age," "the elderly," or "seniors" have been used interchangeably in academic literature, policy, and popular press to refer to people who are aged 55 years or older. Therefore, although we adopt the general term "older adults" in this review, we recognize the extensive heterogeneity among this group of people. Given this diversity, it is important to acknowledge from the outset that older adults are unlikely to respond to stereotypes as a single entity.

The purpose of this review is to report findings on the effects of stereotypes of aging on health outcomes related to older adults, such as physical and mental functioning (specifically) and overall well-being and perceived quality of life (more broadly). The health outcome of focus in each study, or group of studies, has been italicized as the literature is discussed below.

This review first briefly summarizes how stereotypes work to affect health. Then, the literature is organized in terms of the theory and method underpinning each group of studies, with particular focus on stereotype activation experiments. 
The initial discussion will focus primarily (although not exclusively) on the effect of negative stereotypes, while the latter will focus on the effects of positive stereotypes. In doing so, this review highlights that both positive and negative stereotypes of aging can simultaneously have enabling and constraining effects on the actions, performance, decisions, attitudes, and, consequently, holistic health of an older adult.

This review will also highlight a variety of limitations in stereotype research in aging contexts, including a lack of qualitative studies focusing on older adult perspectives and the fluctuating definition of what constitutes "good health" during older age. Increasing our awareness of the multiple effects of stereotypes on individual health outcomes in older adults, and the assumptions embedded in research findings, can promote changes in individual and societal attitudes and behaviors, as well as policy and professional infrastructures that benefit older adults.

\section{How Stereotypes Work to Affect Health}

While physical and cognitive declines in later life can be explained in physiological terms, psychosocial and sociocultural factors also play a key role in this process. Research in mainstream gerontology and psychology has demonstrated the effects of stereotypes of aging on the health of older people; however, the mechanisms by which this occurs is constantly debated within and across disciplines [1013]. Researchers have identified numerous theories of how stereotypes of aging impact older adults, primarily internalization/stereotype embodiment, stereotype threat, downward social comparison/resilience, and (more recently) stereotype boost and upward social comparison/role models [14-16]. Given the range of theories used to explain how stereotypes work to affect health, knowing which theory is driving each study is necessary so that their findings can be clearly understood.

Within these frameworks, experimental studies involving stereotype activation have shown that both implicit (subtle/subconscious) and explicit (conscious) negative age stereotyping can have similar detrimental effects on the performance of older people in physical and mental tasks $[16,17]$. In 2012, Meisner [17] conducted a meta-analysis to compare the strength of positive versus negative age stereotyping effects on behavioral outcomes in older adults. He found, regardless of the type of prime awareness, discipline of study, method, or participant group, that negative age priming elicited a stronger effect (of almost three times larger) on the performance of older individuals during physical and mental tasks than did positive age priming [17]. However, a major limitation of this analysis was that most of the studies included in the review came from Levy's lab. Other reviews, such as Hess et al. [18], found that research on implicit stereotypes has shown that emphasizing positive stereotypes and images of aging can weaken the negative influences of a situation on the behavior of older adults. For example, Hess et al. [19] compared implicit and explicit priming of aging stereotypes on older adults' memory performance and found that those primed implicitly showed significant differences in memory, with positive primes showing greater recall than negative primes. To highlight the heightened effect of domain-specific stereotypes on cognitive and physical outcomes in older adults, Levy and Leifheit-Limson [20, page 230] found that when the age stereotype matched the outcome domain there was a stronger effect on performance, suggesting that when stereotype matching occurs "it is more likely to generate expectations that become self-fulfilling prophecies." They called this the stereotype-matching effect $[20,21]$.

To complicate and extend these findings, however, other studies have shown that explicit positive aging stereotypes can have constraining effects on older people's attitudes and health behavior and that negative aging stereotypes can be resisted by older adults resulting in enabling effects such as feelings of personal empowerment and associated health benefits $[8,15,22,23]$.

Notably, studies on the effects of stereotypes on the health of older individuals typically define health in old age in terms of biomedical and psychosocial models of "successful aging," with indicators of "good health" including low levels (or absence) of disease and disease-related disability, high levels of physical and cognitive functioning, continued social and active engagement in life, and overall contentment, mental health, and the ability to adjust to changes [8]. Nevertheless, the definition of what constitutes "good health" in older age varies across studies. Collectively, research on this topic highlights the complexity in effects of stereotypes on the health of older individuals; there is no conventional pattern as to how stereotypes are internalized into self-stereotypes and/or perceptions of oneself as an older adult, nor the mechanisms underlying this process.

\section{Effects of Negative Stereotypes}

Most studies on stereotypes of aging focus on the constraining effects of negative stereotypes for an older adult's health and ways to minimize/counteract these effects [1]. The findings from stereotype activation experiments and longitudinal studies have been useful in this regard [24].

\subsection{Stereotype Activation and Longitudinal Studies}

3.1.1. Implicit Priming of Stereotypes of Aging (and Stereotype Embodiment/Internalization). Results from numerous experimental studies conducted by Levy and colleagues to test memory, hand writing skills, and walking ability indicated that subliminal exposure to negative age stereotypes can negatively affect (or lead to no changes in) performance in these domains in older adults, while implicit priming with positive stereotypes of aging (such as wise and sage) tended to improve performance [25-27]. For example, in Levy's innovative 1996 study, it was shown that older adults who were implicitly primed with negative aging stereotype words (e.g., senile, dependent, and incompetent) and then asked to undertake memory tasks performed worse than the positively primed group, regardless of age, gender, level of education, previous computer use, mood, and location of residence [27]. Also, Levy et al. [28] and Levy et al. [29] found that aging selfstereotypes had a direct impact on physiological function, 
with negative aging stereotype (subliminal) primes increasing cardiovascular stress in white and African American older individuals, respectively, before and after mental challenges, such as word and math tests.

Moreover, the influence of stereotypes of aging on the health of older individuals can vary across cultures. For example, using a cross-cultural approach, Levy and Langer [30] conducted a comparative study and found that American hearing older adults held the least positive views of the aging process when compared to American deaf older adults and Chinese older adults. Accordingly, the American hearing group performed the worst in memory tasks, followed by the American deaf, and the Chinese group performed the best. However, Yoon et al. [31] did not replicate these findings in their study of Chinese Canadians and Anglophone Canadians because the differences in memory were specific to particular types of memory tests. They also did not conclude that the relationship between culture and aging was mediated by positive attitudes about aging. In spite of these differences, both Levy and Langer and Yoon studies found smaller age differences in those groups who held more positive beliefs about aging. While cross-cultural research regarding stereotypes has shown more positive stereotypes are held in European and Asian cultures, this research has also highlighted that the same number and contexts of negative age stereotypes (i.e., cognitive incompetence) exist in these cultures, similar to Western cultures [32].

Building on Levy's body of work, numerous studies have shown that implicit priming of negative age stereotypes can have adverse effects on physical and cognitive functioning. For example, Auman et al. [33] found stereotype related increases in anxiety, skin conductance, and blood pressure among a sample of middle-aged and older men with hypertension (at an outpatient program) when the prime focused on sickness, helplessness, and dependence. A key finding from this study was that the fear of being perceived as sick (as described in the negative stereotype prime) could actually discourage people from seeking medical assistance, indicating that the concept of stereotype threat (discussed below) is working in conjunction with the internalization of negative stereotypes. In contrast to Levy's [27] findings, however, Stein et al. [34] found that priming with a positive age stereotype did not improve memory performance for a group of older adults. Stein et al. used a photo recall task and a dot location task to measure the memory performance of 60 older adults before and after they were implicitly primed with a negative age stereotype, a positive age stereotype, or neutral primes. It was found that memory performance was undermined among those older adults who were negatively primed and unaware of the primes.

In addition to these short-term effects on performance, Levy et al. [35] showed that aging self-stereotypes can influence older individuals' will-to-live. In this study on responses to hypothetical medical situations, older adults who were subconsciously exposed to negative stereotype primes were less likely to accept medical treatment that could prolong their life. In 2006, Levy et al. [36] examined the age stereotypes of 546 community dwelling adults aged 7096 years and found that those who held more negative and externally (i.e., physical appearance)-related age stereotypes showed greatest hearing loss 36 months after the initial test. Although this study did not use implicit priming, the authors speculated that such findings could have health-behavior consequences, such as older adults not seeking assistance from health professionals due to their belief that hearing loss is a normal and inevitable part of the aging process [36].

Finally, given the limitations of controlled settings in the above studies, more recent research by Levy and colleagues aimed to determine if everyday encounters with negative stereotypes across one's lifetime are associated with cognitive outcomes [37]. They found that, over a 38-year period, individuals with more negative age stereotypes showed significantly worse memory performance compared to those who held less negative age stereotypes and emphasized the robust impact self-relevance has on the effects of stereotypes [37]. This finding supports the view that because older adults are often exposed to negative stereotyping, through everyday interactions in the community and health care settings, this constant exposure may serve as a negative prime which can activate internalized negative age stereotypes [38]. To understand how these internal mechanisms operate alongside external factors in the environment, researchers have drawn on stereotype threat theory with a focus on the effects of explicitly activating negative stereotypes of aging. Stereotype threat theory proposes that presenting familiar negative stereotypes associated with a certain group threatens this group into a scenario where they fear reaffirming these negative depictions [39-42].

\subsubsection{Explicit Priming of Stereotypes of Aging (and Stereotype} Threat). Studies using explicit (rather than implicit) priming techniques have found mostly similar (yet some conflicting) effects of aging stereotypes on physical and cognitive performance, as shown below. Most studies underpinned by stereotype threat theory have examined memory or cognitive performance of older adults [12]. The relationship between stereotype threat and regressions in memory performance has been found among older adults who were explicitly primed with a negative stereotype (in the way the task was framed) [43-45], especially among those who were greatly invested in their memory ability or had high levels of education [46-48]. Stereotype threat has also been found to worsen older adults' math performance [49] and general cognitive ability [50].

A meta-analysis by Horton and colleagues [16] on the impact of stereotype threat on older adults' performance on memory tasks found an overall weighted effect size (i.e., the magnitude of change due to experimental manipulation) of 0.38 (with those exposed to negative stereotype primes in the studies reviewed performing the worst on tasks). However, Horton et al. [16] acknowledged that their review did not include unpublished studies, such as master and doctoral theses, and they cite two such studies that did not find an effect on cognitive performance among older adults exposed to stereotype threat.

A more recent meta-analysis of age-based stereotype threat and effects on performance among older people (average 69.5 years) by Lamont et al. [51] included 22 published and 
10 unpublished articles. They found a significant, yet smallto-medium, effect size of 0.28 (with older adults, regardless of age and gender, memory and cognitive performance were negatively affected by age-based stereotype threat). Lamont et al. also revealed that older adults were less affected by age-based stereotype threat when fact-based (rather than stereotype-based) manipulations are used $(d=0.52)$ and when performance is tested using cognitive (rather than physical) tasks $(d=0.36)$. The inclusion of unpublished studies in this review allowed for a comprehensive analysis of age-based stereotype threat effects which stresses that this threat is a real problem which older people face, especially within formal test contexts. Also, Hess et al. [19], in their comparison of the effects of implicit and explicit priming of aging stereotypes, found that with subtle (as opposed to highly obvious) explicit primes older adults were able to offset the impact of negative stereotypes on memory performance, indicating a type of resilience to the effect of negative stereotypes on performance (a finding that supports resilience theory, discussed below).

Scholl and Sabat [52] argue that to decrease the impact of stereotype threat individuals need to experience a sense of perceived control over the situation which can be achieved by giving older individuals autonomy over personal decisions regarding their health, aiding in the development of more internal health locus of control and motivation to seek out preventative health measures. In addition, given the finding that the effect of stereotype threat (and the associated level of anxiety) is heightened when a person is being tested in a domain where their ability in that area is deeply important to them, "it is vital that practitioners have in-depth knowledge of their clients so as to understand which of their attributes are, and always have been, valued greatly" [52, page 123].

In terms of broader health outcomes related to stereotype threat, Coudin and Alexopoulos [53] examined the effects of explicit negative stereotype activation (i.e., reading a text) on French older adults' self-reported loneliness, risk-taking, subjective health, and help-seeking behavior and found that such activation had harmful effects on participants' selfevaluation and functioning, which may consequently lead to increased dependency. This study highlighted the role of interpersonal and situational factors for inducing dependency among older adults. In support, Burgess et al. [39, page S169] argued that "by recognizing and understanding the factors that can trigger stereotype threat and understanding its consequences in medical settings, providers can prevent it from occurring or ameliorate its consequences for patient behavior and outcomes."

In addition to these short-term performance effects of explicit negative stereotyping, repeated exposure to stereotype threat can result in disengagement from domains that are perceived as threatening to one's self-esteem, which in the longer term can lead to disidentifcation (i.e., avoiding any activities that may result in reaffirming negative stereotypes) $[1,53]$. In the case of older adults, this may involve removing themselves from physical and mental activities because they "no longer view the domain as central to their identity and, as a result, stop expending effort in this domain [39, page S169]," which can have a negative effect on their holistic health [54].
On the other hand (and in line with the Hess et al. [19] study cited above), Horton et al. [40] suggested that some older adults may not be affected by negative stereotypes. This study tested 99 older adults on six dependent measures, namely, memory recall, reaction time, grip strength, flexibility, walking speed, and self-concept. The findings indicated that older adults were affected by the stereotype intervention, but "they suffered no performance decrements on the main dependent measures [40, page 353]." With regard to memory performance, these findings contrast the metaanalysis performed previously by Horton et al. [16] detailed above. Horton et al. [40] note that the specific nature of the participants in their 2010 study (healthy, well educated, and living in a popular retirement locality in Canada) highlights the possibility that certain older adults may be resistant to the potential threat of negative stereotypes of aging. This study showed the importance of understanding how individual older adults respond differently to stereotypes, as well as how they individually perceive their own aging.

3.2. Downward Social Comparison (and Resilience Theory). From a sociological and qualitative perspective, Dionigi and colleagues have found downward social comparison to be typical amongst highly active older adults. Older athletes tend to express a very negative perception of old age or "other" older adults, despite having positive self-perceptions of their own aging [4]. Highly active older adults have been shown to express a desire to maintain physical and mental activity to avoid becoming "old" as they stereotypically understand it (i.e., frail, dependent on others, and diseased) [4, 8]. On one hand, this finding represents an active resistance to aging stereotypes, which can be personally empowering, but on the other hand it reinforces ageism and the individual (and cultural) fear of ill health in old age by positioning ill health in old age as undesirable or irrational, rather than a natural process $[4,23]$.

From a psychosocial perspective, when explaining the acquisition of self-stereotypes of aging, Levy [55] describes the above as the process of identifying oneself as "old" and argues that the more negative the aging stereotypes one holds, the more resistant one is to accepting themselves as old. This resistance could be a form of denial of the physiological realities of the aging process, which could be maladaptive to overall health and a sense of identity in later life $[4,56]$. The important message here is that there is much diversity and complexity in older adults' perceptions and internalization of aging stereotypes, which can affect an individual's health in multiple ways [15].

3.3. Emerging Qualitative Research Findings. Qualitative research findings highlight the complexity inherent in trying to make sense of multidimensional concepts such as perceptions and stereotypes of aging. For example, research by Dionigi et al. [8] found that Canadian women aged 75 years and over who were not physically active expressed more positive attitudes towards their own aging and the term "old" compared to the women in the sample who were highly physically active. In other words, even though these women held high expectations and positive views of their own aging 
and positive stereotypes of old age, it did not mean they were more likely to engage in preventative health behaviors, such as physical activity, in later life. Recently, there has been a call for a more culturally relevant, domain-specific, gender-specific, multidirectional, and multidimensional perspective of aging experiences, feelings, and perceptions [5, 24, 57-60]. With regard to gender differences, a quantitative study by Schafer and Shippee [58] found that feeling older led to more negative views about cognitive aging among women, but not men, aged 55 years and over. More studies on differences between men and women with regard to the effects of stereotypes on health outcomes are needed.

In 2014, Miche et al. [24] used an open-ended, diary approach with 225 men and women (aged 70-88 years) about age-related experiences. They highlighted that content-based, social-cognitive, and social-emotional age-related changes were more important for participants' overall well-being than functional age-related changes. Similarly, a review by Diehl et al. [57] argued that long term health benefits among older people will only be reached if individuals are given the opportunity to take active control of their own lives and develop a clear understanding of their aging process. Diehl et al. developed a conceptual model that allows researchers to create individual need profiles that can be used for intervention and translational research targeting optimal health in later life. These findings question the emphasis in gerontology on the relationship between functional ability and health in later life. They highlight the need to broaden understandings of what constitutes health in old age with particular emphasis on how older individuals define "good health" and "successful aging" in their terms [8].

\section{Effects of Positive Stereotypes}

While it has been shown that implicit and explicit activation of negative age stereotypes can negatively impact older adults' short-term performance in physical and cognitive domains, and potential long-term health outcomes (i.e., health-related behaviors), the effects of positive stereotypes of aging appear more complicated and are comparatively underresearched. The following review will focus on findings that have emerged from experimental studies primarily related to the effects of positive stereotypes of aging on the health of older adults.

4.1. Implicit Priming of Positive Stereotypes of Aging (and Internalization). Preliminary evidence suggests that implicit positive stereotype primes can improve performance in older individuals [16]. For example, many of Levy and colleagues' implicit priming studies reviewed in previous sections revealed that the positively primed group improved their performance in the task being measured, such as memory $[19,27]$, swing time, and balance speed associated with walking [25], or had no change in their performance after being primed, such as a muted cardiovascular response to cognitive challenges [28]. The latter finding led to the assumption that positive primes may act as a buffer to the effects of negative stereotypes [28].

In addition, Levy et al. [35] found in their hypothetical will-to-live study that those who were exposed to positive aging stereotypes were more likely to accept the life-prolonging medical intervention. However, in contrast to Levy's findings on memory [27], it was mentioned earlier that Stein et al. [34] found that older individual's memory performance did not improve after they were primed with a positive age stereotype. Therefore, it cannot be assumed that implicit positive primes will always lead to improved performance in older adults. Nevertheless, researchers have begun to examine if experimentally making people feel younger will result in improvements in physical and mental performance.

4.2. Explicit Priming of Positive Stereotypes of Aging (and Stereotype Boost). Experimental studies on the potential for stereotype boost effects with older adults emerged in 2013. Swift et al. [61] examined the performance of older adults in a domain where they are stereotyped more favorably than younger people (via crossword puzzles). They found that, compared with the control condition, the enhancing social comparison boosted performance among the older adults [61].

Although explicitly making people feel favorable compared to younger people in experimental studies has shown short-term improvements in physical [62] and cognitive [61] tasks, respectively, longer-term effects of explicit positive stereotypes in the daily lives of older adults (such as media messages depicting "healthy, active, and happy" older people) and the effects of dominant "successful aging" discourses (found in academic literature and popular press) are less well known. Making upward, rather than downward, social comparisons has multiple likely effects on the health behaviors of older individuals [63].

4.3. Upward Social Comparison and Role Models. Emerging role model research pertaining to older adults has highlighted the multiple enabling and constraining effects of upward social comparisons on older adults' attitudes and healthrelated behaviors $[15,63,64]$. On the one hand, upward social comparisons, such as comparison with authentic older people who are active and healthy, highlight what is possible in later life which can motivate individuals to become more active themselves and counteract the development of negative selfstereotypes of aging [3]. For example, for already moderately active older adults, older sportspeople may inspire them to become even more active $[15,63,65]$.

On the other hand, if highly active, healthy older people are perceived as unrealistic representations of what is likely in later life, such representations can intimidate others, produce feelings of guilt and anxiety amongst older adults who cannot or do not want to meet that standard, and discourage people from engaging in certain health-related behaviors [3, 64, 65]. For instance, Kotter-Grühn and Hess [22] provided evidence for the negative effects of positive age stereotypes on healthy older adults (such as feeling older and less satisfied with their aging) when confronted with positive and healthy images and descriptions of people their own age. The fact that Hess et al. [46] found a small effect size (0.13) in an explicit positively primed condition on a memory task with older adults lends support to this argument. These findings question the 
assumption that explicitly priming older adults with positive stereotypes will have a positive impact on their performance and overall health; however, these results remain contentious.

Given these contrasting effects of positive stereotypes of aging, and their potential for positive or negative effects on older individual's views of their own aging, and consequently their health or health-related practices, more research is needed on how older adults themselves interpret dominant notions of positive aging. This is particularly important in contemporary Western culture because more positive depictions of aging as a period of ongoing activity and health are emerging [4]. While examples of "exceptional" older adults, such as the athletic feats of older sportspeople, "may be effective in altering societal stereotypes and inspiring those in a younger cohort, they could have a very different effect on those in their own peer group [65, page 131].” More studies using in-depth interviews, life histories, and narrative approaches in gerontology and health care may be effective in understanding how older people react to multiple positive and negative stereotypes. This is because a narrative approach to aging makes ordinary, individual life stories explicit, which can work to breakdown generalizations or stereotypes about aging [66].

\section{Limitations of Stereotype Research in Aging Contexts}

This review of literature on the effects of stereotypes of aging on the health of older adults has highlighted that while valuable knowledge has been gained, such as the finding that both positive and negative stereotypes of aging can be problematic for health, more research in this area is necessary. In particular, the majority of research on this topic (grounded in Levy's work) tends to express a positive-negative binary, or use positive-neutral-negative examples, of aging stereotypes in studies, which can hide the complexity of the effects of stereotypes on older adults [10]. While Levy's work has been invaluable in drawing attention to the effects of stereotypes, such an approach tends to assume that older people interpret stereotypes in similar ways which masks the fluidity of age stereotypes [10].

The findings from studies which test older adults' ability to perform cognitive and/or physical tasks collectively indicate that the testing context, in particular the language used, the expectations set for, and the instructions given to participants in that context, will ultimately influence the results [1, 16]. For example, Hehman and Bugental [67] examined how ageist (patronizing) speech negatively affected older ( $n=69$, ages 61-98) adults' performance (relative to younger people) on a cognitive task and increased their cortisol levels. On the other hand, they found that older adults who had more positive interactions and communication with younger people were not affected by the performance decrements resulting from patronizing communication. Hehman and Bugental argue that, "Repeated exposure to patronizing communication may result in elevated basal cortisol levels, which then has negative implications for older adults' physical, cognitive, and psychological well-being [67, page 558].”
Therefore, reliance on such data that measures older adults' performance is problematic, not only because it provides an inadequate representation of their overall cognitive and/or mental abilities, but also because it can have long-term health implications. This is a major limitation in the interpretation of all stereotype related research within aging populations. Furthermore, researchers, clinicians, or anyone involved in testing older individuals on activities of daily living need to be mindful that "even the subtle cues they give off subconsciously, may be picked up by their participants and ultimately affect how they perform" [16, page 463].

Also, many stereotype activation studies appear to assume that older adults have either a positive or a negative view of aging when in fact both gains and losses are often viewed as part of "healthy aging" in later life $[8,68]$. Furthermore, the assumed definition of what constitutes "good health" in old age in the majority of studies on the effects of aging stereotypes is problematic. A single definition of what constitutes good health in old age does not exist; in fact, in a recent review of "successful aging" literature, Depp et al. [69] found 28 different working definitions of the term across the 27 studies reviewed and Jylhä argued that "there is no single universally agreed definition or direct measure of 'health' or 'health status' [70, page 309]."

Moreover, how an older individual defines health may be quite different from (yet just as valid as) how an "expert" defines health [8]. In other words, “... 'health' belongs to the realm of everyday talk rather than any exact scientific vocabulary [70, page 309]." For instance, Jylhä's paper on self-rated, subjective health highlighted the importance of integrating both cultural and biological information from biomedical and social science disciplines, as well as the individual's bodily sensations and feelings, to make sense of how people rate their health. All in all, "aging is a dynamic process involving both decline and opportunities for continued development [71, page 856]" and stereotypes of aging play a powerful role in this process. Therefore, calls have been made for the use of qualitative research to better understand people's own explanations and accounts of aging and the effects of stereotypes. For example, "Qualitative analyses, inviting people to describe and verbalize their bodily sensations and interpret them in relation to their health status, would help create a new body of information on factors underlying selfrated health [70, page 314].”

Finally, it is important to mention that older adults are often members of multiple stereotyped groups, such as being older, female, and from an ethnic minority or being older, male, and disabled, or being older and from a sexual minority group [72]. Therefore, further research is needed to explore the effects of stereotypes on the health of older adults who belong to more than one stereotyped group. For example, from a psychosocial perspective research could determine if there is a cumulative effect of stereotyping that leads to greater vulnerability or a developed immunity in place that leads to resilience in later life [55]. From a sociological perspective research could examine how intersections of racism, sexism, heterosexism, cultural diversity, and ageism affect the lives of a diverse range of older individuals [73]. 


\section{Summary and Conclusion}

In summary, stereotypes of aging are pervasive in our culture and they have been found to influence how older adults see themselves [22, 74-76]; how older adults view other older adults (social comparison and beliefs about old age) $[8,15$, $22]$; older adults' cognitive and physical performances $[16,25$, $26,28,29,37,55,77]$; the ability of older adults to recover from disease [78]; older adults' health behaviors, including their decisions to engage in cognitive, social, and physical activity $[1,4,21,65,79]$ and/or seek medical assistance $[33,35,53,80]$; as well as how older adults are treated by others and society as a whole $[1,3,4,7,81-83]$.

All of the above outcomes have the potential to affect the holistic health (i.e., mental, physical, social, and emotional well-being) of an older person and ultimately the length and quality of their life. However, to make sense of the studies reporting the complex effects of stereotypes of aging on the health of older individuals, one needs to understand the theoretical underpinnings of the research, the strengths and limitations in the methods used to analyze the effects and recognize the authors' assumed (and perhaps limiting) understanding of what constitutes good health in older age.

\section{Conflict of Interests}

The author declares that there is no conflict of interests regarding the publication of this paper.

\section{References}

[1] S. Horton, J. Baker, and J. M. Deakin, "Stereotypes of aging: their effects on the health of seniors in North American society," Educational Gerontology, vol. 33, no. 12, pp. 1021-1035, 2007.

[2] G. W. Allport, The Nature of Prejudice, Addison-Wesley, Garden City, NY, USA, 1958.

[3] M. Ory, M. K. Hoffman, M. Hawkins, B. Sanner, and R. Mockenhaupt, "Challenging aging stereotypes: strategies for creating a more active society," American Journal of Preventive Medicine, vol. 25, supplement 2, no. 3, pp. 164-171, 2003.

[4] R. A. Dionigi and S. Horton, "The influence of leisure on discourses of aging," in Leisure and Aging: Theory and Practice, $\mathrm{H}$. J. Gibson and J. F. Singleton, Eds., pp. 27-39, Human Kinetics, Champaign, Ill, USA, 2012.

[5] A. E. Kornadt and K. Rothermund, "Contexts of aging: assessing evaluative age stereotypes in different life domains," Journals of Gerontology Series B: Psychological Sciences and Social Sciences, vol. 66 , no. 5, pp. 547-556, 2011.

[6] B. Blytheway, Ageism, Open University Press, Buckingham, UK, 1995.

[7] R. N. Butler, "Age-ism: another form of bigotry," The Gerontologist, vol. 9, no. 4, part 1, pp. 243-246, 1969.

[8] R. A. Dionigi, S. Horton, and J. Bellamy, "Meanings of aging among older Canadian women of varying physical activity levels," Leisure Sciences, vol. 33, no. 5, pp. 402-419, 2011.

[9] O. Jolanki, "Moral argumentation in talk about health and old age," Health, vol. 8, no. 4, pp. 483-503, 2004.

[10] M. L. Hummert, "Commentaries on 'mind matters: cognitive and physical effects of aging self-stereotypes' and author's reply: when is an age stereotype an aging self-stereotype? A commentary," Journals of Gerontology B Psychological Sciences and Social Sciences, vol. 58, no. 4, pp. P212-P213, 2003.

[11] S. C. Wheeler and K. G. DeMarree, "Multiple mechanisms of prime-to-behavior effects," Social and Personality Psychology Compass, vol. 3, no. 4, pp. 566-581, 2009.

[12] S. C. Wheeler and R. E. Petty, "The effects of stereotype activation on behavior: a review of possible mechanisms," Psychological Bulletin, vol. 127, no. 6, pp. 797-826, 2001.

[13] L. A. Zebrowitz, "Aging stereotypes-internalization or inoculation? A commentary," Journals of Gerontology B Psychological Sciences and Social Sciences, vol. 58, no. 4, pp. P214-P215, 2003.

[14] T. Bennett and J. Gaines, "Believing what you hear: the impact of aging stereotypes upon the old," Educational Gerontology, vol. 36, no. 5, pp. 435-445, 2010.

[15] S. Horton, J. Baker, J. Côté, and J. M. Deakin, "Understanding seniors' perceptions and stereotypes of aging," Educational Gerontology, vol. 34, no. 11, pp. 997-1017, 2008.

[16] S. Horton, J. Baker, G. W. Pearce, and J. M. Deakin, "On the malleability of performance: implications for seniors," Journal of Applied Gerontology, vol. 27, no. 4, pp. 446-465, 2008.

[17] B. A. Meisner, "A meta-analysis of positive and negative age stereotype priming effects on behavior among older adults," Journals of Gerontology Series B: Psychological Sciences and Social Sciences, vol. 67, no. 1, pp. 13-17, 2012.

[18] T. M. Hess, J. Birren, and K. Schaie, "Attitudes toward aging and their effects on behavior," in Handbook of the Psychology of Aging, J. E. Birren and K. W. Schaie, Eds., pp. 379-406, Academic Press, San Diego, Calif, USA, 6th edition, 2006.

[19] T. M. Hess, J. T. Hinson, and J. A. Statham, "Explicit and implicit stereotype activation effects on memory: do age and awareness moderate the impact of priming?" Psychology and Aging, vol. 19, no. 3, pp. 495-505, 2004.

[20] B. R. Levy and E. Leifheit-Limson, “The stereotype-matching effect: greater influence on functioning when age stereotypes correspond to outcomes," Psychology and Aging, vol. 24, no. 1, pp. 230-233, 2009.

[21] B. A. Meisner, P. L. Weir, and J. Baker, "The relationship between aging expectations and various modes of physical activity among aging adults," Psychology of Sport and Exercise, vol. 14, no. 4, pp. 569-576, 2013.

[22] D. Kotter-Grühn and T. M. Hess, "The impact of age stereotypes on self-perceptions of aging across the adult lifespan," Journals of Gerontology Series B: Psychological Sciences and Social Sciences, vol. 67, no. 5, pp. 563-571, 2012.

[23] B. Wearing, "Leisure and resistance in an ageing society," Leisure Studies, vol. 14, no. 4, pp. 263-279, 1995.

[24] M. Miche, H.-W. Wahl, M. Diehl, F. Oswald, R. Kaspar, and M. Kolb, "Natural occurrence of subjective aging experiences in community-dwelling older adults," Journals of Gerontology B Psychological Sciences and Social Sciences, vol. 69, no. 2, pp. 174187,2014

[25] J. M. Hausdorff, B. R. Levy, and J. Y. Wei, “The power of ageism on physical function of older persons: reversibility of age-related gait changes," Journal of the American Geriatrics Society, vol. 47, no. 11, pp. 1346-1349, 1999.

[26] B. R. Levy, "Handwriting as a reflection of aging self-stereotypes," Journal of Geriatric Psychiatry, vol. 33, no. 1, pp. 81-94, 2000.

[27] B. R. Levy, "Improving memory in old age through implicit selfstereotyping," Journal of Personality and Social Psychology, vol. 71, no. 6, pp. 1092-1107, 1996. 
[28] B. R. Levy, J. M. Hausdorff, R. Hencke, and J. Y. Wei, "Reducing cardiovascular stress with positive self-stereotypes of aging," Journals of Gerontology B Psychological Sciences and Social Sciences, vol. 55, no. 4, pp. P205-P213, 2000.

[29] B. R. Levy, A. L. Ryall, C. E. Pilver, P. L. Sheridan, J. Y. Wei, and J. M. Hausdorff, "Influence of African American elders' age stereotypes on their cardiovascular response to stress," Anxiety, Stress \& Coping, vol. 21, no. 1, pp. 85-93, 2008.

[30] B. R. Levy and E. Langer, "Aging free from negative stereotypes: successful memory in china and among the American deaf," Journal of Personality and Social Psychology, vol. 66, no. 6, pp. 989-997, 1994.

[31] C. Yoon, L. Hasher, F. Feinberg, T. A. Rahhal, and G. Winocur, "Cross-cultural differences in memory: the role of culture-based stereotypes about aging," Psychology and Aging, vol. 15, no. 4, pp. 694-704, 2000.

[32] T. D. Nelson, "Ageism: prejudice against our feared future self," Journal of Social Issues, vol. 61, no. 2, pp. 207-221, 2005.

[33] C. Auman, H. B. Bosworth, and T. M. Hess, "Effect of healthrelated stereotypes on physiological responses of hypertensive middle-aged and older men," Journals of Gerontology Series B: Psychological Sciences and Social Sciences, vol. 60, no. 1, pp. P3P10, 2005.

[34] R. Stein, F. Blanchard-Fields, and C. Hertzog, "The effects of age-stereotype priming on the memory performance of older adults," Experimental Aging Research, vol. 28, no. 2, pp. 169-181, 2002.

[35] B. R. Levy, O. Ashman, and I. Dror, "To be or not to be: the effects of aging stereotypes on the will to live," Omega, vol. 40, no. 3, pp. 409-420, 1999.

[36] B. R. Levy, M. D. Slade, and T. M. Gill, "Hearing decline predicted by elders' stereotypes," Journals of Gerontology B Psychological Sciences and Social Sciences, vol. 61, no. 2, pp. P82-P87, 2006.

[37] B. R. Levy, A. B. Zonderman, M. D. Slade, and L. Ferrucci, "Memory shaped by age stereotypes over time," Journals of Gerontology B Psychological Sciences and Social Sciences, vol. 67, no. 4, pp. 432-436, 2012.

[38] B. R. Levy, P. H. Chung, and M. Canavan, "Impact of explanatory style and age stereotypes on health across the lifespan," in Handbook of Life-Span Development, K. L. Fingerman, C. A. Berg, J. Smith, and T. C. Antonucci, Eds., pp. 437-456, Springer, New York, NY, USA, 2011.

[39] D. J. Burgess, J. Warren, S. Phelan, J. Dovidio, and M. van Ryn, "Stereotype threat and health disparities: what medical educators and future physicians need to know," Journal of General Internal Medicine, vol. 25, supplement 2, pp. 169-177, 2010.

[40] S. Horton, J. Baker, W. Pearce, and J. M. Deakin, "Immunity to popular stereotypes of aging? seniors and stereotype threat," Educational Gerontology, vol. 36, no. 5, pp. 353-371, 2010.

[41] C. M. Steele, Whistling Vivaldi: And Other Clues to how Stereotypes Affect Us, W.W. Norton \& Company, New York, NY, USA, 2010.

[42] C. M. Steele and J. Aronson, "Stereotype threat and the intellectual test performance of African Americans," Journal of Personality and Social Psychology, vol. 69, no. 5, pp. 797-811, 1995.

[43] A. L. Chasteen, S. Bhattacharyya, M. Horhota, R. Tam, and L. Hasher, "How feelings of stereotype threat influence older adults' memory performance," Experimental Aging Research, vol. 31, no. 3, pp. 235-260, 2005.
[44] O. Desrichard and C. Köpetz, "A threat in the elder: the impact of task-instructions, self-efficacy and performance expectations on memory performance in the elderly," European Journal of Social Psychology, vol. 35, no. 4, pp. 537-552, 2005.

[45] T. A. Rahhal, L. Hasher, and S. J. Colcombe, "Instructional manipulations and age differences in memory: now you see them, now you don't," Psychology and Aging, vol. 16, no. 4, pp. 697706, 2001.

[46] T. M. Hess, C. Auman, S. J. Colcombe, and T. A. Rahhal, “The impact of stereotype threat on age differences in memory performance," Journals of Gerontology B Psychological Sciences and Social Sciences, vol. 58, no. 1, pp. P3-P11, 2003.

[47] T. M. Hess, J. T. Hinson, and E. A. Hodges, "Moderators of and mechanisms underlying stereotype threat effects on older adults' memory performance," Experimental Aging Research, vol. 35, no. 2, pp. 153-177, 2009.

[48] S. K. Kang and A. Chasteen, "The moderating role of agegroup identification and perceived threat on stereotype threat among older adults," International Journal of Aging and Human Development, vol. 69, no. 3, pp. 201-220, 2009.

[49] D. Abrams, R. J. Crisp, S. Marques, E. Fagg, L. Bedford, and D. Provias, "Threat inoculation: experienced and imagined intergenerational contact prevents stereotype threat effects on older people's math performance," Psychology and Aging, vol. 23, no. 4, pp. 934-939, 2008.

[50] D. Abrams, A. Eller, and J. Bryant, "An age apart: the effects of intergenerational contact and stereotype threat on performance and intergroup bias," Psychology and Aging, vol. 21, no. 4, pp. 691-702, 2006.

[51] R. A. Lamont, H. J. Swift, and D. Abrams, "A review and metaanalysis of age-based stereotype threat: negative stereotypes, not facts, do the damage," Psychology and Aging, vol. 30, no. 1, pp. 180-193, 2015.

[52] J. M. Scholl and S. R. Sabat, "Stereotypes, stereotype threat and ageing: implications for the understanding and treatment of people with Alzheimer's disease," Ageing \& Society, vol. 28, no. 1, pp. 103-130, 2008.

[53] G. Coudin and T. Alexopoulos, “'Help me! I’m old!' How negative aging stereotypes create dependency among older adults," Aging \& Mental Health, vol. 14, no. 5, pp. 516-523, 2010.

[54] S. K. Whitbourne and J. R. Sneed, “The paradox of well-being, identity process, and stereotype threat: ageism and its potential relationships to the self in later life," in Ageism: Stereotyping and Prejudice Against Older Persons, T. D. Nelson, Ed., pp. 247-274, MIT Press, Cambridge, Mass, USA, 2002.

[55] B. R. Levy, "Mind matters: cognitive and physical effects of aging self-stereotypes," Journals of Gerontology Series B: Psychological Sciences and Social Sciences, vol. 58, no. 4, pp. P203-P211, 2003.

[56] B. R. Levy and M. R. Banaji, "Implicit ageism," in Ageism: Stereotypes and Prejudice against Older Persons, T. Nelson, Ed., pp. 4975, MIT Press, Cambridge, Mass, USA, 2002.

[57] M. Diehl, H.-W. Wahl, A. E. Barrett et al., "Awareness of aging: theoretical considerations on an emerging concept," Developmental Review, vol. 34, no. 2, pp. 93-113, 2014.

[58] M. H. Schafer and T. P. Shippee, "Age identity, gender, and perceptions of decline: does feeling older lead to pessimistic dispositions about cognitive aging?" Journals of Gerontology Series B: Psychological Sciences and Social Sciences, vol. 65, no. 1, pp. 91-96, 2010.

[59] G. J. Westerhof and A. E. Barrett, "Age identity and subjective well-being: a comparison of the United States and Germany," 
Journals of Gerontology B Psychological Sciences and Social Sciences, vol. 60, no. 3, pp. S129-S136, 2005.

[60] G. J. Westerhof, S. K. Whitbourne, and G. P. Freeman, "The aging self in a cultural context: the relation of conceptions of aging to identity processes and self-esteem in the United States and the Netherlands," Journals of Gerontology Series B: Psychological Sciences and Social Sciences, vol. 67, no. 1, pp. 52-60, 2012.

[61] H. J. Swift, D. Abrams, and S. Marques, "Threat or boost? Social comparison affects older people's performance differently depending on task domain," Journals of Gerontology B Psychological Sciences and Social Sciences, vol. 68, no. 1, pp. 23-30, 2013.

[62] Y. Stephan, A. Chalabaev, D. Kotter-Grühn, and A. Jaconelli, "Feeling younger, being stronger': an experimental study of subjective age and physical functioning among older adults," Journals of Gerontology Series B: Psychological Sciences and Social Sciences, vol. 68, no. 1, pp. 1-7, 2013.

[63] S. Horton, R. A. Dionigi, and J. Bellamy, "Women aged 75 and over: attitudes towards health-related role models and female masters athletes," The International Journal of Interdisciplinary Social and Community Studies, vol. 7, no. 3, pp. 33-47, 2013.

[64] P. Lockwood, A. L. Chasteen, and C. Wong, "Age and regulatory focus determine preferences for health-related role models," Psychology and Aging, vol. 20, no. 3, pp. 376-389, 2005.

[65] S. Horton, "Masters athletes as role models? Combating stereotypes of aging," in The Masters Athlete: Understanding the Role of Sport and Exercise in Optimizing Aging, J. Baker, S. Horton, and P. L. Weir, Eds., pp. 122-136, Routledge, London, UK, 2010.

[66] G. Kenyon, E. Bohlmeijer, and W. L. Randall, Eds., Storying in Later Life: Issues, Investigations, and Interventions, Oxford University Press, New York, NY, USA, 2011.

[67] J. A. Hehman and D. B. Bugental, "Responses to patronizing communication and factors that attenuate those responses," Psychology and Aging, vol. 30, no. 3, pp. 552-560, 2015.

[68] S. Wurm, C. Tesch-Römer, and M. J. Tomasik, "Longitudinal findings on aging-related cognitions, control beliefs, and health in later life," Journals of Gerontology Series B: Psychological Sciences and Social Sciences, vol. 62, no. 3, pp. P156-P164, 2007.

[69] C. Depp, I. V. Vahia, and D. Jeste, "Successful aging: focus on cognitive and emotional health," Annual Review of Clinical Psychology, vol. 6, pp. 527-550, 2010.

[70] M. Jylhä, "What is self-rated health and why does it predict mortality? Towards a unified conceptual model," Social Science and Medicine, vol. 69, no. 3, pp. 307-316, 2009.

[71] M. M. Davis, L. A. Bond, A. Howard, and C. A. Sarkisian, "Primary care clinician expectations regarding aging," The Gerontologist, vol. 51, no. 6, pp. 856-866, 2011.

[72] D. Padgett, "Aging minority women," in Gender, Culture, and Ethnicity: Current Research about Women and Men, L. A. Peplau, S. C. DeBro, R. C. Veniegas, and P. L. Taylor, Eds., pp. 173-181, Mayfield, Mountain View, Calif, USA, 1999.

[73] S. E. Mock, S. M. Shaw, E. M. Hummel, and C. Bakker, "Leisure and diversity in later life: ethnicity, gender, and sexual orientation," in Leisure and Aging: Theory and Practice, H. J. Gibson and J. F. Singleton, Eds., pp. 111-125, Human Kinetics, Champaign, Ill, USA, 2012.

[74] B. R. Levy and L. M. Myers, "Preventive health behaviors influenced by self-perceptions of aging," Preventive Medicine, vol. 39, no. 3, pp. 625-629, 2004.

[75] B. R. Levy, M. D. Slade, and S. V. Kasl, "Longitudinal benefit of positive self-perceptions of aging on functional health," Journals of Gerontology B Psychological Sciences and Social Sciences, vol. 57, no. 5, pp. P409-P417, 2002.
[76] B. R. Levy, M. D. Slade, S. R. Kunkel, and S. V. Kasl, "Longevity increased by positive self-perceptions of aging," Journal of Personality and Social Psychology, vol. 83, no. 2, pp. 261-270, 2002.

[77] B. R. Levy, "Stereotype embodiment: a psychosocial approach to aging," Current Directions in Psychological Science, vol. 18, no. 6, pp. 332-336, 2009.

[78] B. R. Levy, M. D. Slade, J. May, and E. A. Caracciolo, "Physical recovery after acute myocardial infarction: positive age selfstereotypes as a resource," International Journal of Aging and Human Development, vol. 62, no. 4, pp. 285-301, 2006.

[79] C. A. Sarkisian, T. R. Prohaska, M. D. Wong, S. Hirsch, and C. M. Mangione, "The relationship between expectations for aging and physical activity among older adults," Journal of General Internal Medicine, vol. 20, no. 10, pp. 911-915, 2005.

[80] B. A. Meisner and J. Baker, "An exploratory analysis of aging expectations and health care behavior among aging adults," Psychology and Aging, vol. 28, no. 1, pp. 99-104, 2013.

[81] L. D. Grant, "Effects of ageism on individual and health care providers' responses to healthy aging," Health and Social Work, vol. 21, no. 1, pp. 9-15, 1996.

[82] T. Nelson, Ed., Ageism: Stereotyping and Prejeduce Against Older Persons, MIT Press, Cambridge, Mass, USA, 2004.

[83] T. M. Nemmers, "The influence of ageism and ageist stereotypes on the elderly," Physical and Occupational Therapy in Geriatrics, vol. 22, no. 4, pp. 11-20, 2004. 


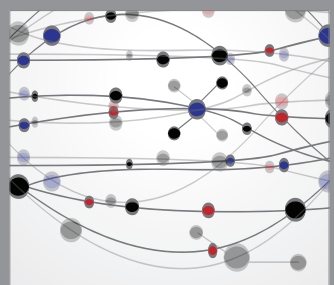

The Scientific World Journal
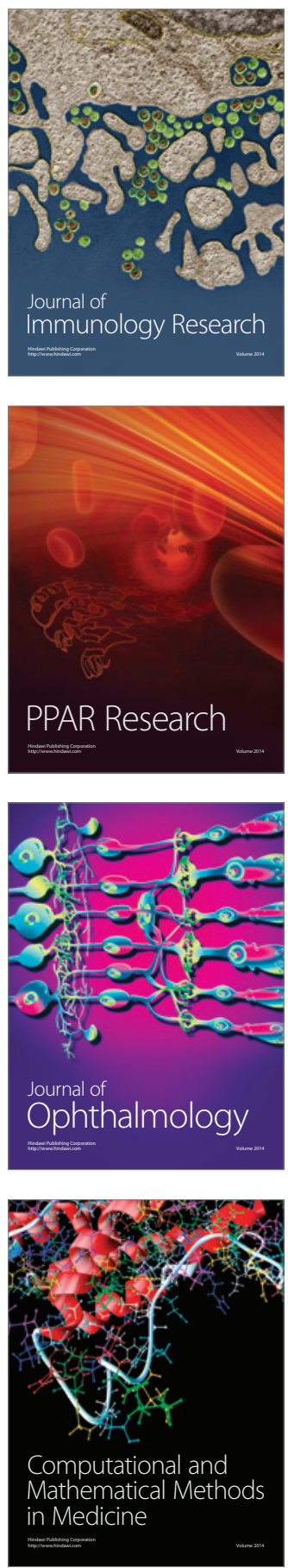

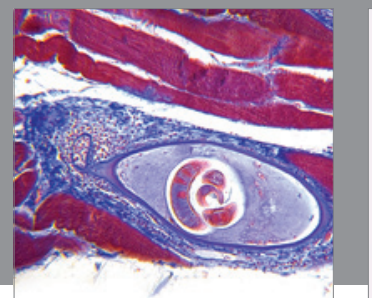

Gastroenterology

Research and Practice
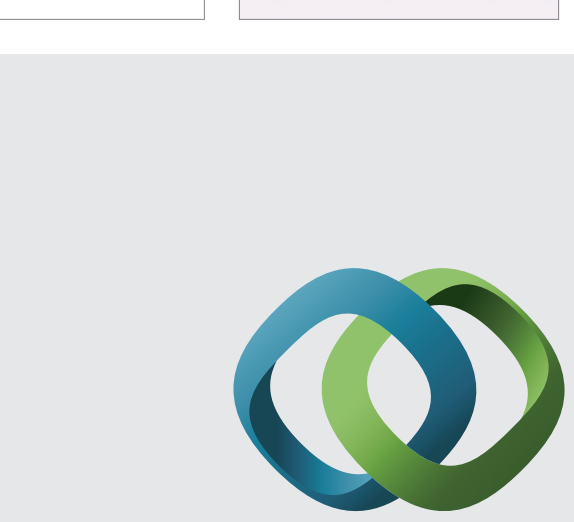

\section{Hindawi}

Submit your manuscripts at

http://www.hindawi.com
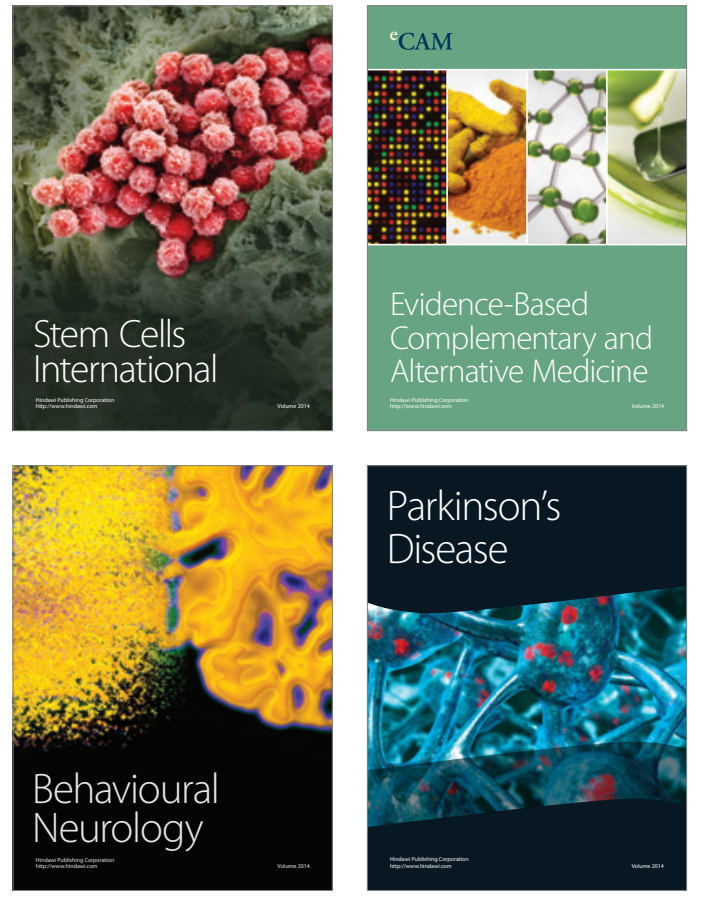
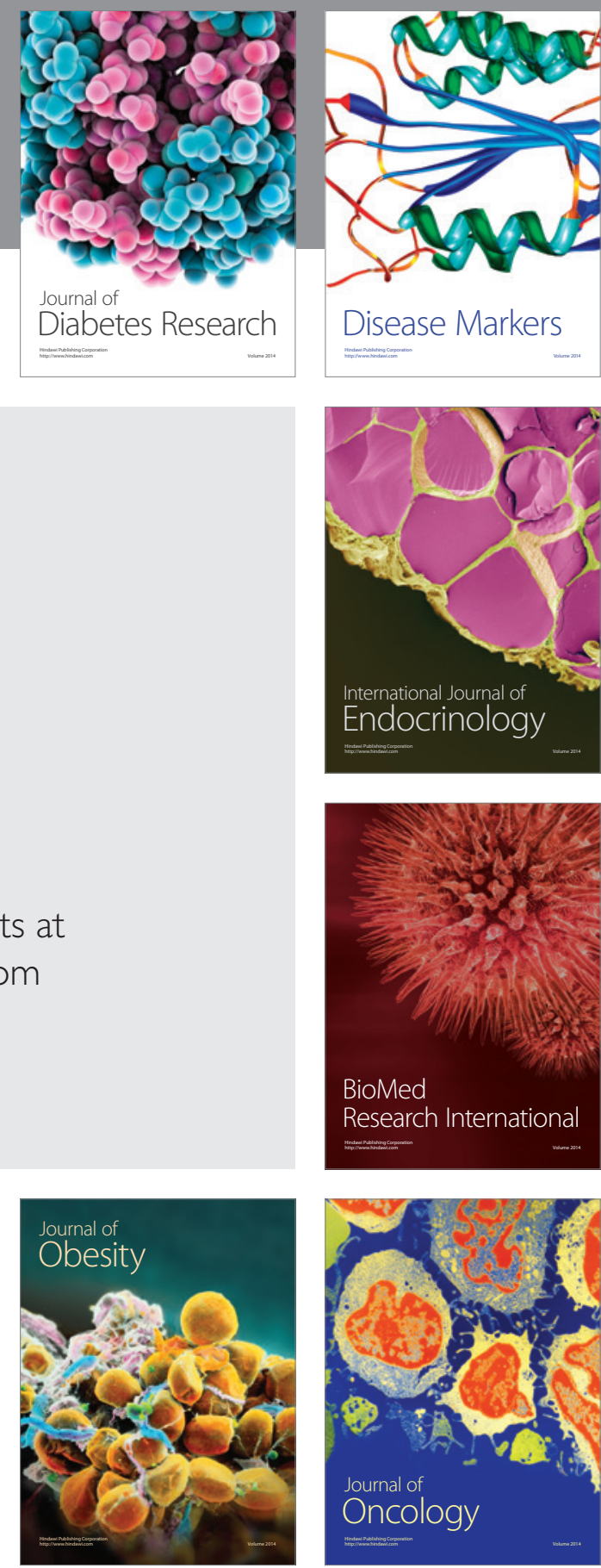

Disease Markers
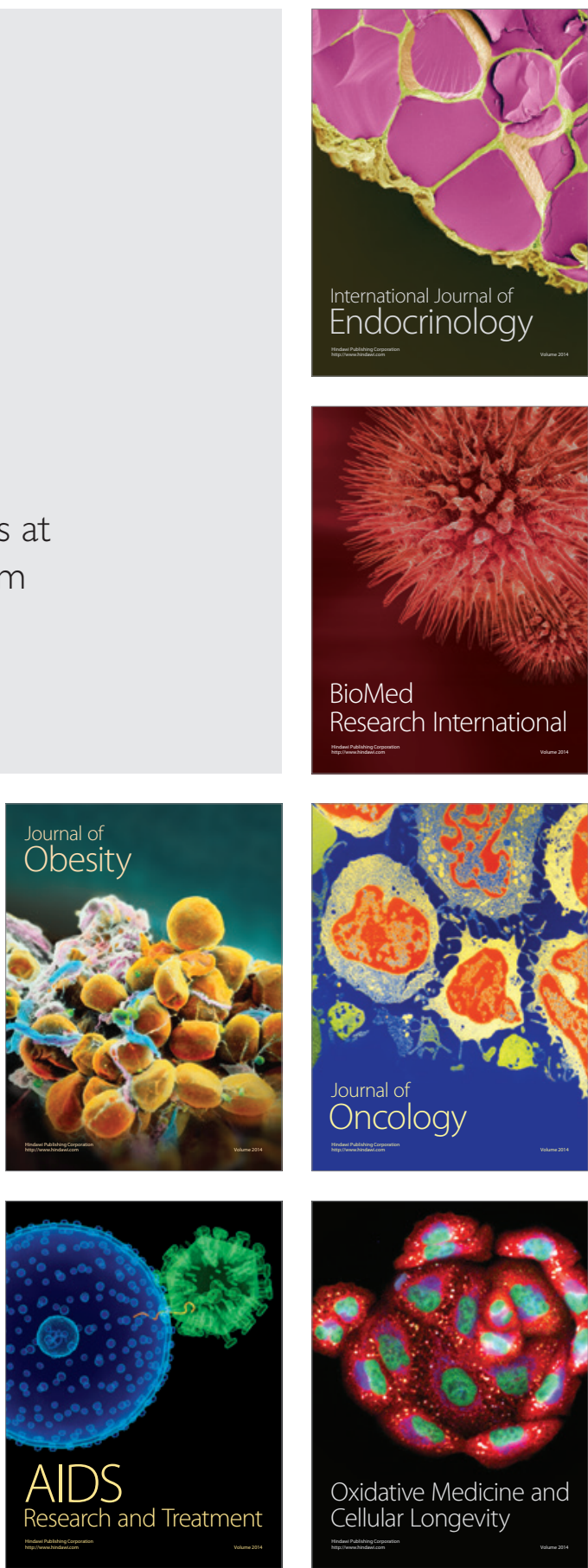